\title{
Field dynamics of coherent synchrotron radiation using a direct numerical solution of Maxwell's equations
}

\author{
A. Novokhatski* \\ SLAC National Accelerator Laboratory, Menlo Park, California 94025, USA
}

(Received 18 March 2011; published 21 June 2011)

\begin{abstract}
We present and discuss the properties of the coherent electromagnetic fields of a very short, ultrarelativistic bunch in a rectangular vacuum chamber inside a bending magnet. The analysis is based on the results of a direct numerical solution of Maxwell's equations together with Newton's equations. We use a new dispersion-free time-domain algorithm which employs a more efficient use of finite element mesh techniques and, hence, produces self-consistent and stable solutions for very short bunches. We investigate the fine structure of the coherent synchrotron radiation fields. We also discuss coherent edge radiation. We present a clear picture of the field using the electric field lines constructed from the numerical solutions. This method should be useful in the study of existing and future concepts of particle accelerators and ultrafast coherent light sources, where high peak currents and very short bunches are envisioned.
\end{abstract}

DOI: 10.1103/PhysRevSTAB.14.060707

PACS numbers: 29.27.Bd, 29.20.db, 41.75.Ht, 41.60.Ap

\section{INTRODUCTION}

The coherent synchrotron radiation (CSR) fields have a strong action on the beam dynamics of very short bunches, which are moving in the bends of all kinds of magnetic elements. They are responsible for additional energy loss and energy spread; microbunching and beam emittance growth. These fields may bound the efficiency of damping rings, electron-positron colliders, and ultrafast coherent light sources, where high peak currents and very short bunches are envisioned. This is relevant to most highbrightness beam applications. On the other hand, these fields together with transition radiation fields can be used for beam diagnostics or even as a powerful resource of $\mathrm{THz}$ radiation.

A history of the study of CSR and a good collection of references can be found in [1] and also in the well-known papers [2-4]. The physics of the coherent fields of a bunch rotating in a circle is very well understood. Steady state analytic solutions, which have been found, describe the essential properties of the CSR fields. However, a steady state approach along with other assumptions restrict these formulas for practical applications. By other assumptions, we mean that a beam is considered to be rigid, radiation occurs either in free space or between parallel plates, and there are no transient effects or coherent edge radiation. So for practical applications one has to rely on numerical solutions. Electromagnetic theory suggests several meth-

\footnotetext{
*novo@slac.stanford.edu
}

Published by the American Physical Society under the terms of the Creative Commons Attribution 3.0 License. Further distribution of this work must maintain attribution to the author(s) and the published article's title, journal citation, and DOI. ods on how to calculate CSR fields. The most popular method is to use Lienard-Wiechert potentials. Another approach is to solve numerically the approximate equations, which are a Schrodinger-type equation. These numerical methods are very well described in [5-8]. However, these methods still have several assumptions and do not give a full description of the CSR fields. We suggest that a direct solution of Maxwell's equations together with Newton's equations can describe the detailed structure of the CSR fields.

There are a lot of finite-difference schemes, which numerically solve Maxwell's equations since the first one was published in 1966 [9]. Most of them are so-called "explicit" schemes, which means that the value of the field at the new time step is calculated only by the field values from the previous time step. Stability conditions for these schemes do not allow a time step to be greater than or equal to a space (mesh) step. This limitation brings an additional troublesome effect for wavelengths that are compared to a mesh step. We state that this effect works like a frequency dispersion media, which is "hidden" in the finitedifference equation.

We propose to use an implicit method, which is free of dispersion in the longitudinal direction. The method has been used to solve the wake potential problem of the very short bunches [10]. A further development of this methodology is to increase its capability for modeling coherent radiation.

\section{METHOD}

The main strategy of the method is to use an implicit scheme for the field calculation; Fourier expansion in the vertical direction, a traveling mesh, and an ensemble of particles for a bunch dynamics calculation. 


\section{A. Equations}

We will solve numerically Maxwell's and Newton's equations for an ultrarelativistic bunch of charged particles moving in a rectangular vacuum chamber inside a bending magnet. Electromagnetic components $\vec{E}, \vec{B}$ must satisfy the equations

$$
\begin{aligned}
\frac{1}{c} \frac{\partial}{\partial t} \vec{E} & =\nabla \times \vec{B}-\frac{4 \pi}{c} \vec{J}_{b} & \nabla \cdot \vec{E} & =4 \pi \rho_{b} \\
\vec{E}_{\text {wall }} \times \vec{n} & =0 & \frac{1}{c} \frac{\partial}{\partial t} \vec{B} & =-\nabla \times \vec{E} \\
\nabla \cdot \vec{B} & =0 & \vec{B}_{\text {wall }} \cdot \vec{n} & =0 .
\end{aligned}
$$

A charge density $\rho_{b}$ and a charge current $\vec{J}_{b}$ must satisfy a continuity equation

$$
\nabla \cdot \vec{J}_{b}+\frac{1}{c} \frac{\partial}{\partial t} \rho_{b}=0 \quad \rho_{b}=\sum_{k} \rho_{k}(\vec{x}) \vec{J}_{b}=\sum_{k} \rho_{k}(\vec{x}) \vec{v}_{k}
$$

A Newton force $\vec{F}$ includes electromagnetic components and a bending magnetic field $\vec{B}_{\text {bend }}$ :

$$
\begin{aligned}
\frac{\partial}{\partial t} \vec{p}_{k} & =\vec{F}=e \vec{E}+\frac{\vec{v}_{k}}{c} \times e\left(\vec{B}+\vec{B}_{\text {bend }}\right) \\
\vec{p}_{k} & =\frac{m \vec{v}_{k}}{\sqrt{1-\frac{v_{k}^{2}}{c^{2}}}}
\end{aligned}
$$

\section{B. Implicit scheme and wakefield calculations}

Modeling ultrafast phenomena requires a special algorithm for solving the electromagnetic equations. This algorithm must be free of frequency dispersion which means that all propagating waves must have their natural phase velocity, completely independent of the simulation parameters like a mesh size or a time step. We suggest an implicit algorithm which does not have stability issues and employs a more efficient use of finite element mesh techniques. This method can produce self-consistent stable solutions for very short bunches. The scheme could have dispersion in the transverse direction. However, electromagnetic fields, which interact with a beam, propagate in the vacuum chamber at small angles, so the effect of dispersion in the transverse direction is less important than dispersion in the longitudinal direction.

We have already used this same approach for wakefield calculations. An implicit, dispersion-free time-domain algorithm has been used in the computer code designed in 1976 for wakefield dynamics studies at the Novosibirsk Electron-Positron Linear Collider VLEPP [11]. At that time we managed to calculate wakefields of a $1.8 \mathrm{~mm}$ bunch. Later this algorithm has been used to solve the wake potential problem of much shorter bunches and has proved to be quite powerful in describing the wakefields at the TESLA Liner Collider $(\sigma=0.7 \mathrm{~mm})[12]$ and TESLA

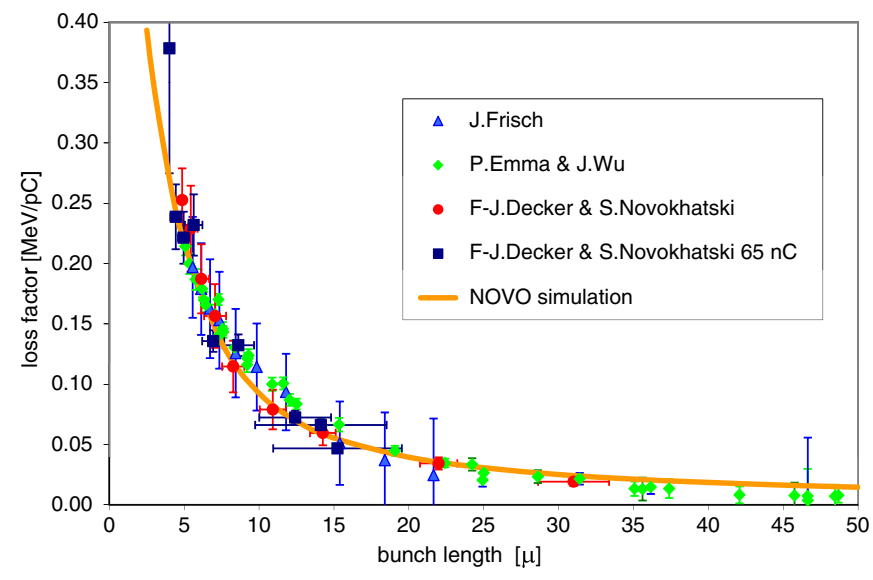

FIG. 1. Measured and simulated loss factor of the transport line (LTU) and undulator vacuum chamber of LCLS. Symbols correspond to the results of different measurements. The continuous dark yellow line shows the numerical result.

Test Facility FEL $(\sigma=25 \mu)$ [13]. Recently, we got an opportunity to make a comparison with wakefield measurements at Linac Coherent Light Source (LCLS) for a transport line [linac to undulator (LTU)] and undulator vacuum chamber. Results of a computation of the wakefield loss factor of a several micron long bunch $(\sigma=4 \mu)$ showed very good agreement with the measurement results [14]. Here we present this comparison in Fig. 1, which includes results of several measurements. The continuous dark yellow line shows the numerical result. The implicit method has been also very efficiently used in the numerical solution of the Vlasov and the Fokker-Plank equation for the longitudinal beam dynamics in a damping ring $[15,16]$.

To employ the implicit scheme, we transform Eqs. (1) to the second order equations:

$$
\begin{aligned}
\frac{1}{c^{2}} \frac{\partial^{2}}{\partial t^{2}} \vec{E} & =\nabla^{2} \vec{E}-4 \pi\left(\frac{1}{c^{2}} \frac{\partial}{\partial t} \vec{J}_{b}+\nabla \cdot \rho_{b}\right) \\
\frac{1}{c^{2}} \frac{\partial^{2}}{\partial t^{2}} \vec{B} & =\nabla^{2} \vec{B}+\nabla \times \vec{J}_{b} .
\end{aligned}
$$

Formulas for the numerical approximation of an equation of second order are given in [17]. In this publication we also present an analysis and comparison of the explicit and implicit schemes with application to CSR field calculations. Additional details can be found in [18].

\section{Traveling mesh and bunch particles}

To decrease the amount of needed memory we use a traveling mesh. This is very important for bunch compressor simulations at higher beam energies where the bunch length is a micron but the distance between bends is tens of meters. The mesh moves with the speed of light and we can definitely assume that the electromagnetic field in front of the bunch is zero. Because the time delay due to the bending magnet in the chicane is very small, we do not 
need more space for the bunch. A traveling mesh does not change the accuracy of the scheme or any conditions of stability.

To simulate the real shape of a nonmonochromatic bunch moving, for example, in a bending magnet, we will use an ensemble of particles. We will track each particle and average the current (particle velocities) over the mesh. The charge density distribution will be integrated using the continuity equation for charge and current. This will help to smooth out errors of particle transitions from one cell to another. The real reason for the better accuracy is that the time step multiplied by the speed of light is equal to the mesh step in the longitudinal direction.

To check this method, we made a comparison with results of the one-dimensional analytical approach and we found good agreement in some simple cases. However, we have found a much more interesting detailed structure of the CSR fields, which has not been described by any previous study. We will present a clear picture of the field in the form of electric force lines and verify the method from a physical point of view.

\section{CSR FIELD DYNAMICS}

\section{A. Radiation in a bend}

Initially we will try to understand how a bunch field remakes itself when a bunch is rotated in a magnetic field. We have calculated the electromagnetic field of a threedimensional Gaussian bunch initially moves along the vacuum chamber very close to the speed of light. At some point the bunch enters a vertical magnetic field of a bend. In these simulations we use relative units. The ratio of the bunch length to a bending radius is $\sigma / \rho=$ $3 \times 10^{-3}$, the horizontal size is half the bunch length $\sigma_{x} / \sigma=0.5$. We did not find any strong dependence on vertical size as long as it is smaller than the bunch length. The horizontal vacuum chamber size is $a=0.5 \rho$ and vertical chamber size is $h=0.1 \rho$. The number of Fourier modes is mainly determined by the longitudinal bunch size and the chamber height. In our examples it is around 10. There are no singularities in our simulations. A bunch has a finite size in all three dimensions. We consider only an ultrarelativistic case, when the transverse force of the electric field of the bunch is almost compensated by the transverse force of the bunch magnetic field, so a particle vertical velocity is negligible in comparison with the horizontal and longitudinal velocities. In order to show the field line distribution, we increase the vertical vacuum chamber size and the vertical bunch size to make field planar, i.e., to make two-dimensional field line plots.

Figure 2 shows snapshots of the electric field lines at different time moments. The white boxes show bunch contours. Red arrows show the direction of a bunch velocity. Before entering a bend the bunch has only a transverse field, which can be seen as a set of vertical lines. A new field that is generated in a bend is a set of ovals, which
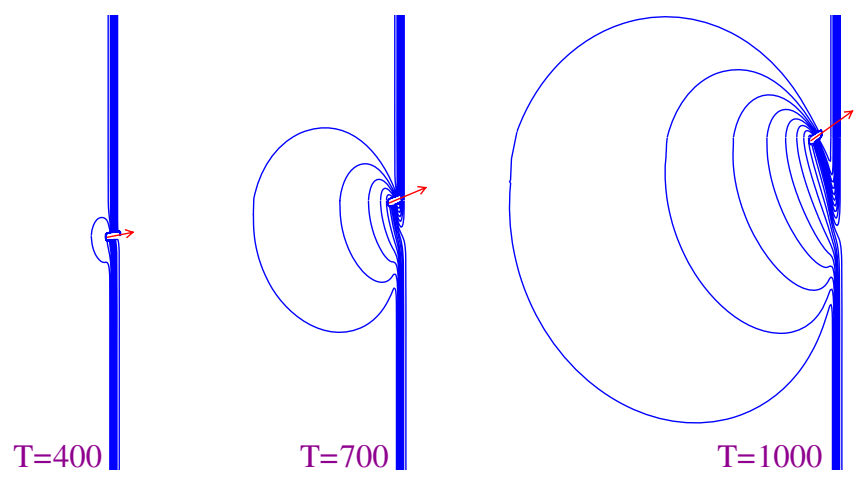

FIG. 2. Snapshots of electric field lines of a bunch, which is moving in a magnetic field. White boxes show the bunch contour. Red arrows show the directions of the bunch velocity.

increase in size with a time. We can outline two time periods of the field formation. The first is when a bunch is moving inside the region of its initial transverse field. The first two plots in Fig. 2 are related to this first period. The second period starts when the bunch is delayed so much that it is out of the region of the initial transverse field. The last plot in Fig. 2 shows this situation. The transverse field continues to move straight with the speed of light, so we may consider it to be the field of the edge radiation in a bend.

A more detailed picture of the field lines is shown in Fig. 3, where green arrows show the directions of the electric field.

One can see that the upper field lines take the position of the lower lines and a part of the lower field lines take the position of the upper lines. However, at the far ends the transverse field lines continue traveling in the same initial direction. It is easy to explain such behavior if we present this field as a sum of two fields:

$$
\vec{E}=\vec{E}_{\mathrm{dp}}+\vec{E}_{\mathrm{in}} \quad \vec{B}=\vec{B}_{\mathrm{dp}}+\vec{B}_{\mathrm{in}} .
$$

The first field $\vec{E}_{\mathrm{dp}}, \vec{B}_{\mathrm{dp}}$ is the field of a dipole, which consists of two oppositely charged bunches. One bunch

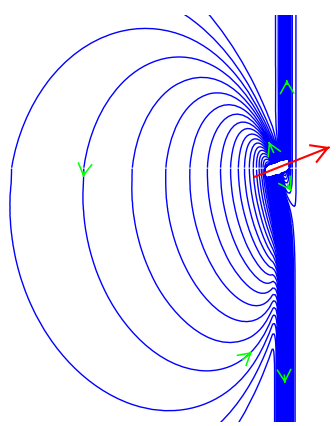

FIG. 3. Detailed structure of the field pattern. The red arrow shows the direction of the bunch velocity. Green arrows show the field line direction. Upper field lines take the position of the lower lines and a part of the lower field lines take the position of the upper lines. 


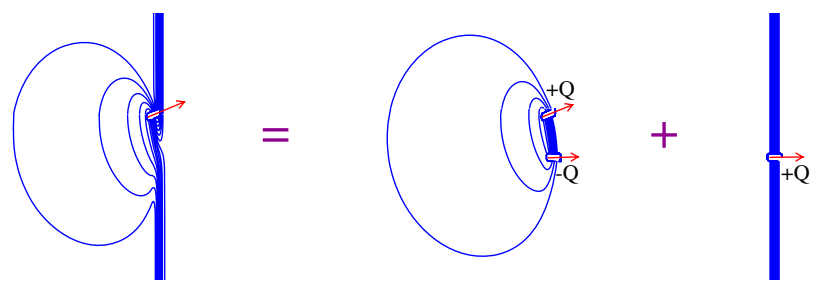

FIG. 4. Decomposition of the field of a bunch moving in a magnetic field (left plot) into two fields: a field of a dipole (middle plot) and a field of a bunch moving straight in the initial direction (right plot). Red arrows show directions of a bunch velocity.

is the "real" one with a positive charge. This bunch is rotated in the magnetic field while the other bunch is a "virtual" one, which has an opposite charge and travels straight in the initial direction. The second field $\vec{E}_{\text {in }}, \vec{B}_{\text {in }}$ is the field of another virtual, but positively charged bunch, which travels straight along the initial direction. Naturally the virtual bunches together sum to zero. This decomposition is shown in Fig. 4.

There could be a close analogy between the field decomposition and the Feynman diagram shown in Fig. 5. A real electron produces a virtual photon, which decays into an electron-positron pair, corresponding to a dipole. The positron can annihilate with the ongoing scattered electron to emit a photon. This photon corresponds to synchrotron radiation.

We note that this decomposition can also help to improve the accuracy of the numerical calculation of the force acting on the bunch particles because we remove the strong initial bunch field. We decompose the fields just for the purpose of showing the structure of the CSR field. This model is good for a straight beam pipe until a bunch meets the chamber wall.

A dense set of field lines in Fig. 3 also reveals a fine structure of the field in front of a bunch. This $\gamma$-type region is common in a classical synchrotron radiation. There are several publications on the electric field patterns of the synchrotron radiation [19-21]. We chose Ref. [20], as it supplies a picture of the field lines of a particle with a relativistic factor $\gamma=6$. The characteristic wavelength of

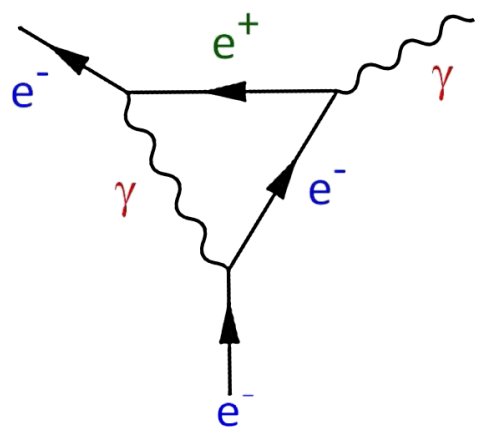

FIG. 5. Decomposition as an analog of the Feynman diagram. the synchrotron radiation or an equivalent value of the bunch length for this relativistic factor is $\sigma=R / \gamma^{3}$, which is very close to our bunch length. Figure 6 shows this finite structure together with a plot from Ref. [20]. The plots are rotated in order to have the velocities in the same direction for a better comparison. Plots are also scaled in order to have approximately the same bending radius. We can state that the $\gamma$ region before a bunch is very close for both cases. As was mentioned above, we can distinguish two stages of the field formation. Now we can separate the interaction with the field $\vec{E}_{\text {in }}, \vec{B}_{\text {in }}$ and the field $\vec{E}_{\text {dp }}$, $\vec{B}_{\text {dp }}$. The interaction with the field $\vec{E}_{\text {in }}, \vec{B}_{\text {in }}$ continues only for the time when a bunch is inside the region of this transverse field. We can estimate the distance $D^{+}$(or equivalent time) when a bunch leaves this region. This means that the bunch delay $\delta$ must be more than the bunch length $2 \sigma$ :

$$
\delta=\rho \varphi-\rho \sin \varphi \approx \rho \frac{\varphi^{3}}{3} \geq 2 \sigma .
$$

From this relation we have

$$
D^{+}=\rho \varphi \geq\left(6 \rho^{2} \sigma\right)^{1 / 3} .
$$

The distance $D^{+}$is $37 \%$ less than a characteristic overtaking length $L_{0}=2\left(3 \sigma \rho^{2}\right)^{1 / 3}$ according to [3]. The transverse field is located only near the bunch, in the region, which can be approximated by the bunch size $\sigma_{x}$ or $\sigma_{y}$ or the bunch length $\sigma$. A bunch leaves the transverse field much earlier when his transverse displacement $\Delta x$ exceeds the field region,

$$
\Delta x=\frac{1}{2} \rho \varphi^{2} \geq \sigma,
$$

so the distance $D^{+}$is

$$
D^{+}=\rho \varphi \geq \sqrt{2 \rho \sigma} .
$$

The distribution of the electric field $\vec{E}_{\text {in }}$ on the horizontal plane in the vertical center of the vacuum chamber and the
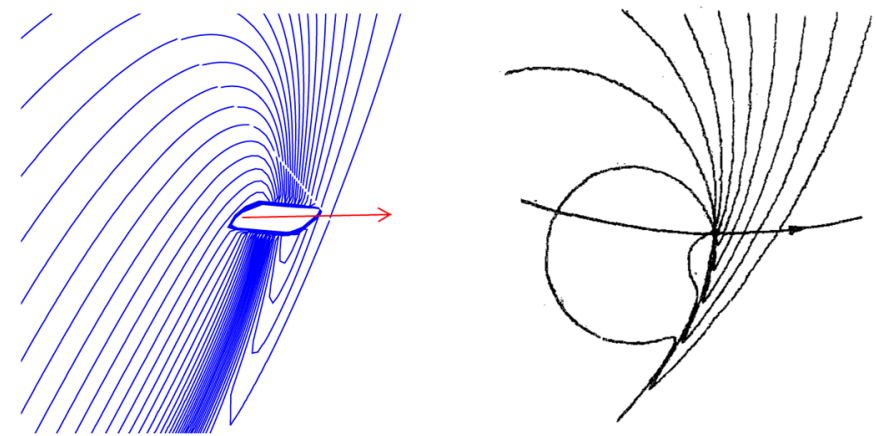

FIG. 6. Fine structure of the field pattern in front of a bunch. The left plot shows field lines near a bunch. The right plot presents a picture from Ref. [20] for $\gamma=6$. The plots are rotated and scaled in order to have the same direction for the velocities and approximately the same bending radius. 


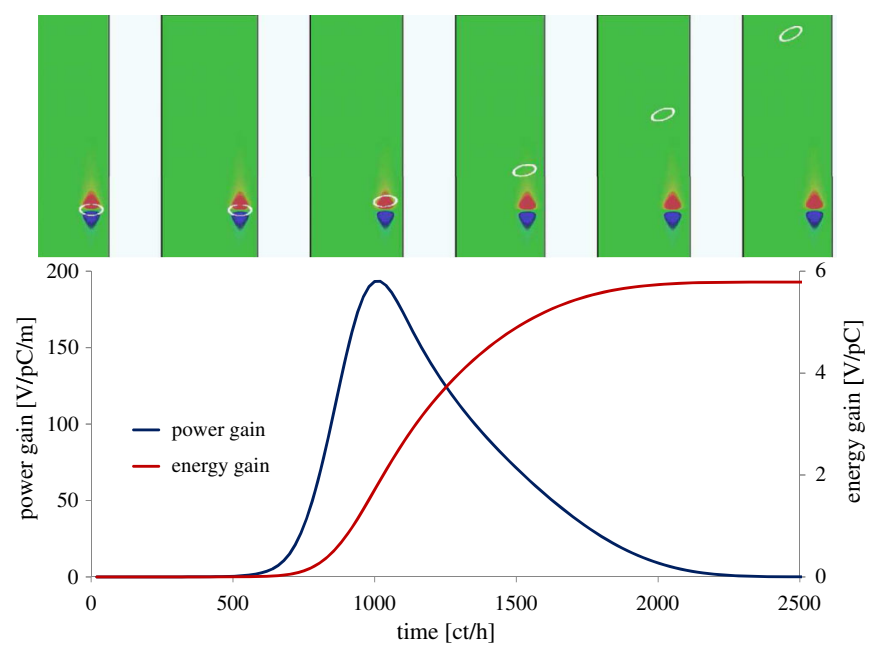

FIG. 7. The electric field distribution on the horizontal plane in the vertical center of the vacuum chamber and a bunch position at different times. The red color corresponds to a high positive value of the field and the blue color corresponds to a low negative value of the field. The white oval shows the real bunch contour. Coherent power and energy gain are shown by a blue and a red curve.

bunch contour at different time steps are shown in Fig. 7. The red color corresponds to a high positive value of the field and the blue color corresponds to a low negative value of the field. The white oval shows the real bunch contour. The oval covers approximately $90 \%$ of the bunch. The plot also shows the coherent power loss $P$ (gain in our case), which was calculated using the formula

$$
P=\int\left(\vec{J}_{b} \cdot \vec{E}\right) d x d y d z
$$

and the energy loss $W$ (gain), which is just a time integral of the power loss

$$
W=\int P d t
$$

The power and energy gain of a bunch in the field $\vec{E}_{\text {in }}$ was calculated for a bunch length of $0.6 \mathrm{~mm}$. The maximum power is achieved at the time when the bunch is in the red region of the field. The interaction of the bunch with the field $\vec{E}_{\mathrm{dp}}$ continues for a much longer time. Figure 8 shows the absolute value of the electric field on the horizontal plane in the vertical center of the vacuum chamber in consecutive time steps. The white oval shows the real bunch contour. When a dipole is created, an electric field appears between a real bunch and a virtual bunch. This field increases in value and reaches a maximum value when the bunches are completely separated and then it goes down as bunches move apart leaving fields only around the bunches. The bunch acquires an energy loss while interacting with the field $\vec{E}_{\mathrm{dp}}$. To study the fields acting on the particles inside the bunch, we calculated
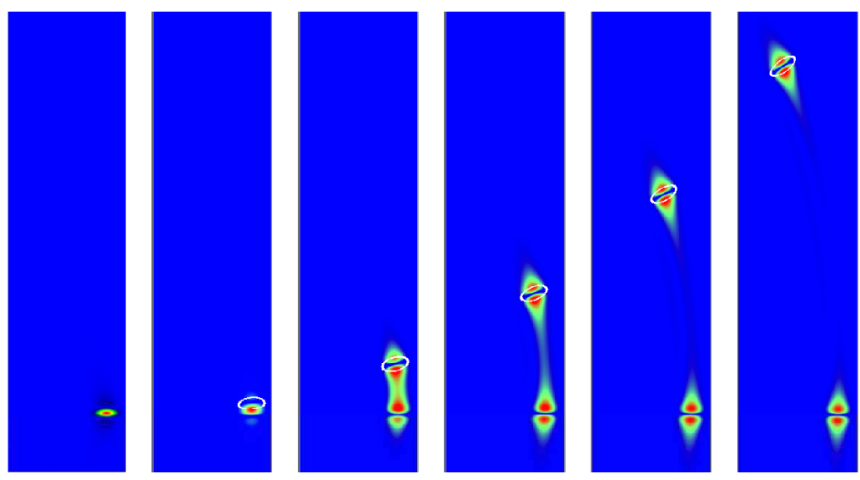

FIG. 8. Absolute value of the electric field $\vec{E}_{\mathrm{dp}}$ on the horizontal plane in the vertical center of the vacuum chamber in consecutive time steps. The red color corresponds to maximum value. The blue color corresponds to the minimum value of the field. The white oval shows the real bunch contour.

the distribution of a collinear force $F_{\|}$and a transverse force $F_{\perp}$ :

$$
F_{\|}=\vec{J}_{b} \cdot \vec{E} \quad F_{\perp}=\left(\vec{J}_{b} \times \vec{E}\right)_{x} .
$$

Now we include both fields $\vec{E}_{\text {in }}$ and $\vec{E}_{\mathrm{dp}}$ in the electric field $\vec{E}$. We have found some very exciting fine structure of the force acting on the particle in the bunch. Figure 9 show a distribution of forces on the horizontal plane in the vertical center of the vacuum chamber at three time moments. The left three vertical plots in Fig. 9 show a bunch charge distribution. The starting plots are at the bottom at the time when a bunch just enters the magnetic field. Red arrows show the direction of the bunch velocity. The middle three vertical plots show a transverse force. Again, the red arrows show the direction of the force.
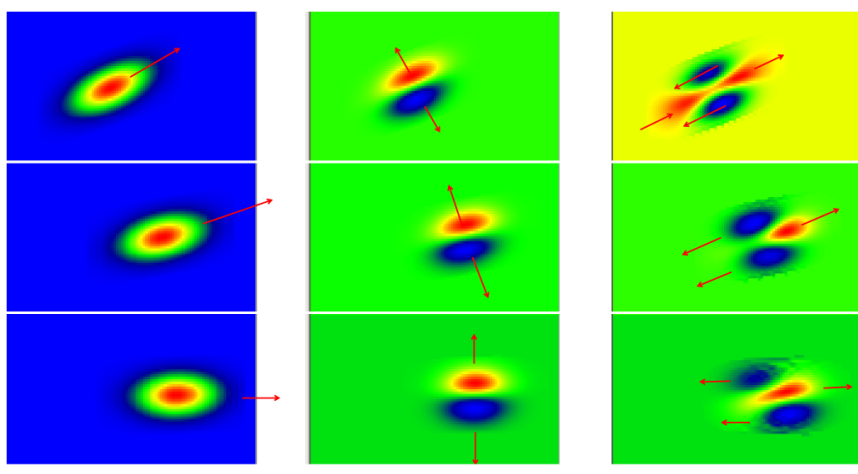

FIG. 9. Bunch charge distribution, transverse forces, and collinear forces on the horizontal plane in the vertical center of the vacuum chamber at three time moments. The starting plots are at the bottom at the time when a bunch just enters the magnetic field. The left three vertical plots show a bunch charge distribution. The red arrows show the direction of the bunch velocity. The middle three vertical plots show a transverse force. Again, the red arrows show the direction of the force. The right three vertical plots show the collinear force, which is responsible for an energy gain or energy loss. 

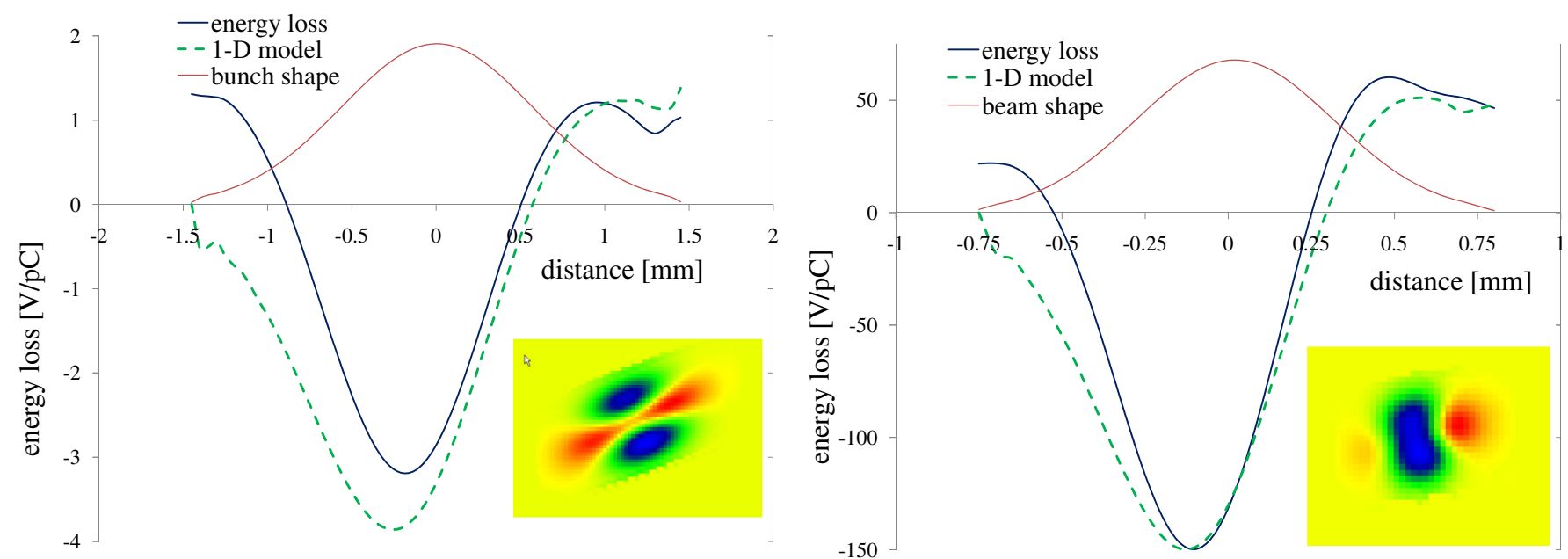

FIG. 10. Integrated energy loss along the transverse direction as a function of the longitudinal coordinate for two values of bending radius. The left plot corresponds to the upper plot at the right in Fig. 8. The right plot shows the result for a bending radius 40 times larger and a bunch length that is 2 times smaller. The green dashed line is from the analytical 1D model.

The transverse force is the well-known space-charge force, which probably is compensated by a magnetic force in the ultrarelativistic case. The right three vertical plots show the collinear force, which is responsible for an energy gain or an energy loss. The red color corresponds to acceleration and energy gain and the blue color corresponds to deceleration and energy loss. The red arrows are collinear or anticollinear with a bunch velocity. We did not include these forces in the beam dynamics simulation in order to make the physical picture clear.

We see here that the forces on the bunch are very complicated. The particles, which are in the center of the bunch, in front of the bunch and at the end are accelerating, whereas the particles at the boundaries are decelerating. This means that a bunch gets an additional energy spread in the transverse direction. The total effect is deceleration and the bunch loses energy. The asymmetry of the longitudinal fields can also be seen in Fig. 2, which shows the electric field line distributions. The bunch shape deformation due to the difference in the angular velocity along the radial position is usually small and can be seen only after some time; however, the ultrasmall beam emittance can be changed.

The integrated energy loss along the transverse direction as a function of the longitudinal coordinate is shown in Fig. 10 together with a bunch longitudinal distribution.
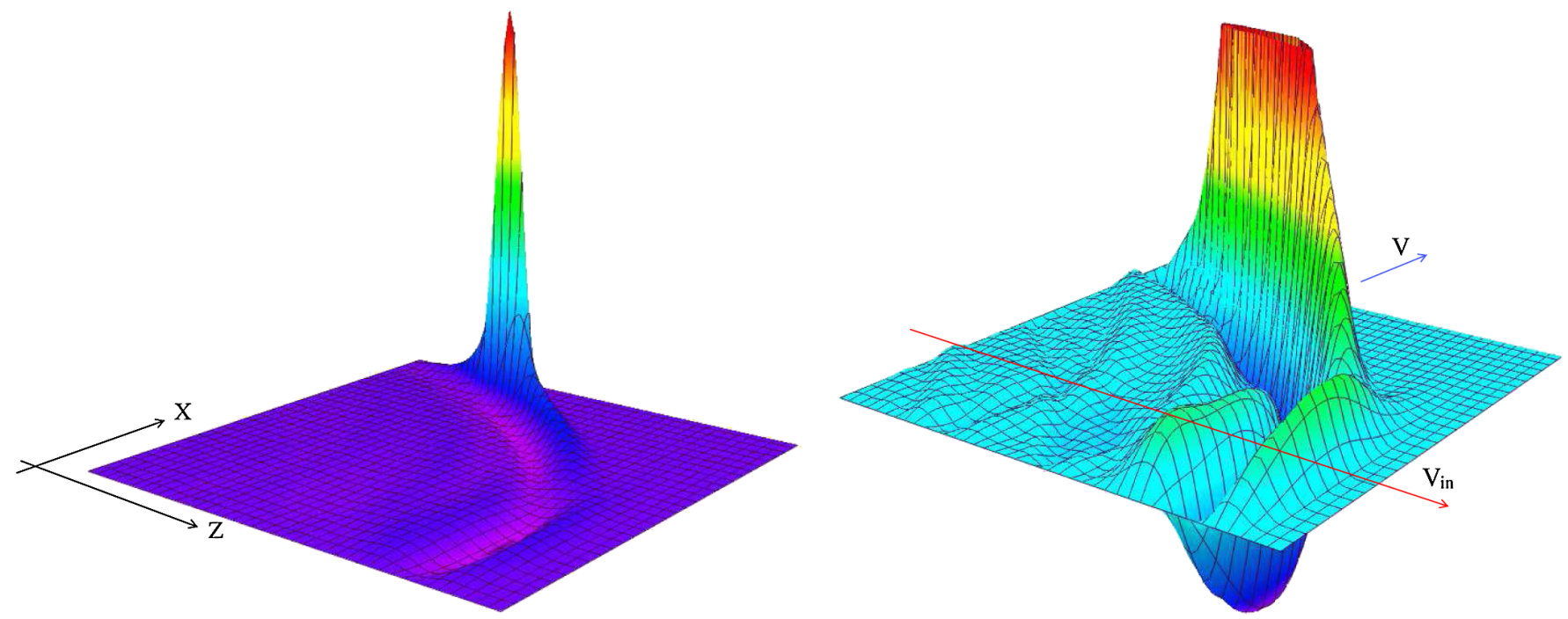

FIG. 11. Coherent edge radiation. Distribution of the magnetic field on the horizontal plane in the vertical middle of the vacuum chamber. The large peak corresponds to the bunch field. The right picture is a magnified image of the left picture. Note the scales in the $\mathrm{X}$ and $\mathrm{Z}$ directions are different. A red arrow shows the initial bunch $\mathrm{X}$ position and the direction of the bunch velocity. A blue arrow shows the direction of the bunch velocity at this time. 


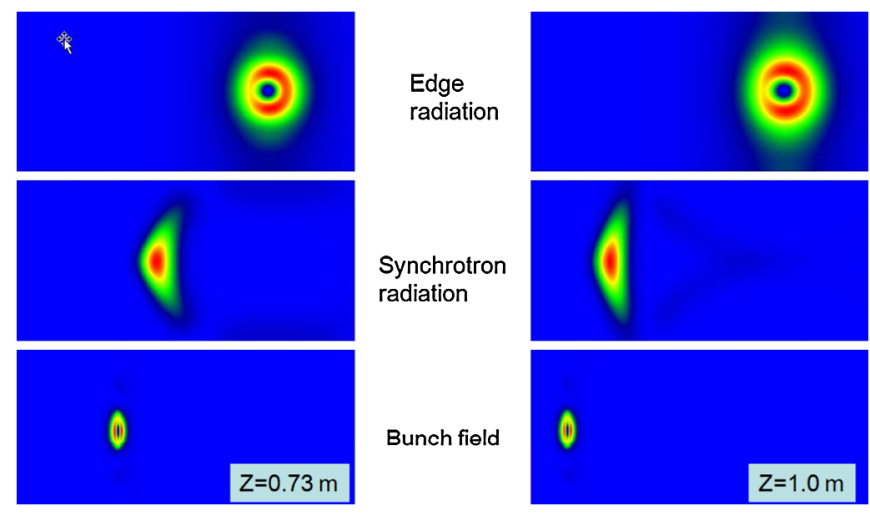

FIG. 12. Images of radiation in the form transverse magnetic field distributions. The left and the right sets of vertical plots correspond to different longitudinal positions. Each plot in a set shows a distribution at a different time. First comes an image of edge radiation, then the image of synchrotron radiation, and finally a bunch field image.

One can see that the head of a bunch and tail are accelerated, when the rest of the bunch is decelerated. The shape of the energy loss distribution is compared with the analytical 1D model [3] including shielding (green dashed line). We obtain a better agreement with the shape of the energy loss distribution for a larger bending radius and smaller bunch length. This comparison is shown at the right plot of Fig. 10. The transverse energy spread is smaller for a larger bending radius. This complicated structure of the collinear field is very important. A bunch will get an additional transverse energy spread, which cannot be compensated. This energy spread in the magnetic field immediately generates emittance growth. This effect can limit the efficiency of the magnetic bunch compressors and as a result the efficiency of FELs.

\section{B. Coherent edge radiation}

As we mention above, an ultrarelativistic bunch and CSR fields are moving together and interact for a long time. However one can see a field, which propagates straight ahead from the initial beam $\mathrm{X}$ position. This field can be seen very well when the bunch gets a large horizontal displacement. Figure 11 shows the distribution of the magnetic field on the horizontal plane in the vertical middle of the vacuum chamber. The large peak corresponds to the bunch field. The right picture is a magnified image of the left picture. Note the scales in the $\mathrm{X}$ and $\mathrm{Z}$ directions are different. A red arrow shows the initial bunch $\mathrm{X}$ position and the direction of the bunch velocity. A blue arrow shows the direction of the bunch velocity at this time.

Figure 12 shows images of the coherent radiation in the form of transverse magnetic field distributions on the vertical planes of the vacuum chamber. The left and the right sets of vertical plots correspond to different longitudinal positions. Each plot in a set shows a distribution at a different time. At first we see an image of edge radiation, then the image of synchrotron radiation, and finally a bunch field image.

The calculated images of the coherent edge radiation look very similar to the images, which we have seen on the YAG screen after the dump magnets, which bend down the beam at LCLS.

\section{CONCLUSIONS}

We have developed a new method for the numerical solution of Maxwell's equations. We can analyze the fine structure of the coherent synchrotron fields, excited by a short bunch in a bending magnet. We present a clear picture of the field in the form of electric force lines. We found good agreement with an analytical one-dimensional model in simple cases. However, we have found much more interesting and detailed structure of the CSR fields, which have not been described by any previous study. A very important result is discovering the structure of the complicated collinear force. A bunch will get an additional energy spread in the transverse direction from the collinear force. This immediately leads to an emittance growth and decoherence that could limit FEL lasing for very short bunches. This effect needs more study.

\section{ACKNOWLEDGMENTS}

The author would like to thank Mike Sullivan and R. Clive Field for help and valuable comments, Franz-Josef Decker, Paul J. Emma, and Yunhai Cai for support and interest in this work, and physicists of the Beam Physics Department for useful discussions. This work was supported by Department of Energy Contract No. DE-AC03$76 \mathrm{SF} 00515$.

[1] J. B. Murphy, in ICFA Beam Dynamics Newsletter (2004), No. 35, p. 20 [http://icfa-usa.jlab.org/archive/newsletter/ icfa_bd_nl_35.pdf].

[2] J. S. Nodvick and D. S. Saxon, Phys. Rev. 96, 180 (1954).

[3] Ya.S. Derbenev et al., TESLA FEL-Report No. 1995-05, 1995.

[4] E.L. Saldin et al., Nucl. Instrum. Methods Phys. Res., Sect. A 398, 373 (1997).

[5] R. Li, Nucl. Instrum. Methods Phys. Res., Sect. A 429, 310 (1999).

[6] G. Bassi et al., Nucl. Instrum. Methods Phys. Res., Sect. A 557, 189 (2006).

[7] M. Borland, Phys. Rev. ST Accel. Beams 4, 070701 (2001).

[8] G. V. Stupakov and I. A. Kotelnikov, Phys. Rev. ST Accel. Beams 12, 104401 (2009).

[9] K. S. Yee, IEEE Trans. Antennas Propag. 14, 302 (1966).

[10] A. Novokhatski, Report No. SLAC-PUB-11556, 2005.

[11] V. Balakin et al., in Proceedings of the All Union Accelerator Conference, Dubna, 1978, p. 143 (in 
Russian). Translated to English: Report No. SLAC TRANS-188, 1978.

[12] A. Novokhatski and A. Mosnier, Report No. DAPNIA/ SEA-96-08, 1996.

[13] R. Brinkmann et al., in Proceedings of the European Particle Accelerator Conference, Vienna, 2000 (EPS, Geneva, 2000).

[14] A. Novokhatski, in Proceedings of the 31st International Free Electron Laser Conference (FEL 09), Liverpool, UK (STFC Daresbury Laboratory, Warrington, 2009).

[15] A. Novokhatski, Report No. SLAC-PUB-11251, 2005.
[16] A. Novokhatski and T. Weiland, in Proceedings of the European Particle Accelerator Conference, Vienna, 2000 (Ref. [13]).

[17] A. Novokhatski and M. Sullivan, in Proceedings of IPAC'10, Kyoto, Japan, TUPEB028 [http://accelconf .web.cern.ch/AccelConf/IPAC10/papers/tupeb028.pdf].

[18] A. Novokhatski, in Proceedings of 2011 Particle Accelerator Conference, New York, NY, USA (2010).

[19] R. Y. Tsien, Am. J. Phys. 40, 46 (1972)

[20] S. G. Arutyunyan, Sov. Phys. Usp. 29, 1053 (1986).

[21] T. Shintake, Nucl. Instrum. Methods Phys. Res., Sect. A 507, 89 (2003). 